\title{
Trans-tail regulation-mediated suppression of cryptic transcription
}

\author{
Jungmin Choi $\mathbb{D}^{1 凶}$, Zae Young Ryoo², Dong-Hyung Cho ${ }^{2}$, Hyun-Shik Lee ${ }^{2}$ and Hong-Yeoul Ryu ${ }^{\circledR}$
}

(c) The Author(s) 2021

Crosstalk between post-translational modifications of histone proteins influences the regulation of chromatin structure and gene expression. Among such crosstalk pathways, the best-characterized example is $\mathrm{H} 2 \mathrm{~B}$ monoubiquitination-mediated $\mathrm{H} 3 \mathrm{~K} 4$ and $\mathrm{H} 3 \mathrm{~K} 79$ methylation, which is referred to as trans-tail regulation. Although many studies have investigated the fragmentary effects of this pathway on silencing and transcription, its ultimate contribution to transcriptional control has remained unclear. Recent advances in molecular techniques and genomics have, however, revealed that the trans-tail crosstalk is linked to a more diverse cascade of histone modifications and has various functions in cotranscriptional processes. Furthermore, H2B monoubiquitination sequentially facilitates H3K4 dimethylation and histone sumoylation, thereby providing a binding platform for recruiting Set3 complex proteins, including two histone deacetylases, to restrict cryptic transcription from gene bodies. The removal of both ubiquitin and SUMO, small ubiquitin-like modifier, modifications from histones also facilitates a change in the phosphorylation pattern of the RNA polymerase II C-terminal domain that is required for subsequent transcriptional elongation. Therefore, this review describes recent findings regarding trans-tail regulation-driven processes to elaborate on their contribution to maintaining transcriptional fidelity.

Experimental \& Molecular Medicine (2021) 53:1683-1688; https://doi.org/10.1038/s12276-021-00711-x

\section{INTRODUCTION}

In eukaryotic organisms, nuclear DNA is compressed into a highorder packaged structure referred to as chromatin, which comprises repeating building blocks called nucleosomes ${ }^{1}$. Each nucleosome is made up of $\sim 147$ bp of DNA wrapped around an octamer of histone proteins containing two copies of each of histones $\mathrm{H} 2 \mathrm{~A}, \mathrm{H} 2 \mathrm{~B}, \mathrm{H} 3$, and $\mathrm{H} 4$. Subsequently, histones can then undergo several types of post-translational modifications, including methylation at arginine (R), phosphorylation at serine $(S)$ and threonine $(T)$, and other diverse types of modifications (acetylation, methylation, ubiquitylation, sumoylation, biotinylation, and ADP-ribosylation) at the lysine (K) region ${ }^{2,3}$. However, studies have shown that these modifications alter interactions between DNA and histones, thereby allowing the recruitment of chromatinmodifying enzymes and transcription factors ${ }^{4}$.

Histone modifications can also modulate the establishment of other modifications within the same histone (in cis) or in a different histone (in trans), thereby providing crosstalk among the histones ${ }^{5}$. Such crosstalk generates complex signals that facilitate or repress chromatin-mediated processes. For instance, in humans, phosphorylation at $\mathrm{H} 3 \mathrm{~S} 10$ and acetylation at $\mathrm{H} 4 \mathrm{~K} 16$ act cooperatively to recruit the P-TEFb (positive transcriptional elongation factor b) to the nucleosome, thus promoting transcriptional elongation ${ }^{6}$. Additionally, Chk1-mediated H3T11 phosphorylation allows the acetylation of H3K14 by Gon 5 for the transcriptional activation of genes encoding products involved in cell cycle regulation. However, DNA damage drives this crosstalk in the reverse direction to induce transcriptional repression?
A study further showed that the yeast SAGA (Spt-Ada-Gcn5acetyltransferase) complex stimulated acetylation on nucleosomes containing methylated H3K4 via the tandem Tudor domains of the Sgf29 subunit ${ }^{8}$, and this histone acetylation conversely provoked Set1-driven H3K4 methylation ${ }^{9}$.

The best-characterized histone crosstalk occurs between H2B monoubiquitination (ub)-dependent H3K4 and K79 methylation, which is an evolutionarily conserved trans-tail pathway, to maintain dynamic chromatin structure during transcription ${ }^{10,11}$. Recent reports have revealed that this traditional crosstalk is regulated in a more complex manner and is involved in more diverse functions than others have reported (Fig. 1). A study showed that H2BK123 ub-stimulated H3K4 di-(me2), but not tri(me3) methylation promoted histone sumoylation, thereby providing a binding site for the Set3 HDAC (histone deacetylase) complex ${ }^{12}$. This Set3 complex-mediated histone deacetylation in the $5^{\prime}$ ORF region was then reported to facilitate the suppression of spurious transcriptional initiation in genes ${ }^{13,14}$. On the basis of these presented facts, this review provides an overview of newly discovered functions of the trans-histone H3K4 methylation process regulated by $\mathrm{H} 2 \mathrm{Bub}$ in restricting cryptic transcription.

\section{Intragenic cryptic transcription}

Transcription is a complex process that requires the sequential assembly of many factors, including chromatin-modifying and remodeling enzymes that act to elongate the RNA polymerase machinery on nucleosomal templates ${ }^{15,16}$. During transcription, the chromatin structure must be dynamically modified and

\footnotetext{
${ }^{1}$ Department of Biomedical Sciences, Korea University College of Medicine, Seoul 02841, Republic of Korea. ${ }^{2}$ BK21 FOUR KNU Creative BioResearch Group, School of Life Sciences, College of National Sciences, Kyungpook National University, Daegu 41566, Republic of Korea. ${ }^{凶}$ email: jungminchoi@korea.ac.kr; rhr4757@knu.ac.kr
} 


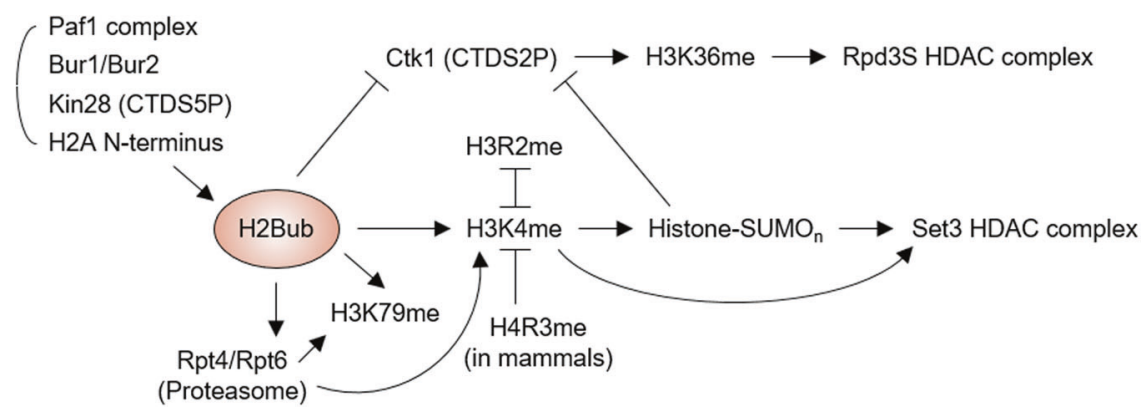

Fig. 1 H2B monoubiquitination-centered crosstalk pathway in S. cerevisiae. The Paf1 complex, Bur1/Bur2 and Kin28 kinases, and $\mathrm{N}$-terminal tails of $\mathrm{H} 2 \mathrm{~A}$ facilitate $\mathrm{H} 2 \mathrm{~B}$ monoubiquitination (ub) during yeast transcription. $\mathrm{H} 2 \mathrm{Bub}$ then promotes $\mathrm{H} 3 \mathrm{~K} 4$ and $\mathrm{H} 3 \mathrm{~K} 79$ methylation (me) both directly and via the proteasomal ATPases Rpt4 and Rpt6. H3R2me mutually antagonizes H3K4me, whereas H4R3me interferes with the binding ability of H3K4 methyltransferase (no reports of H4R3me in S. cerevisiae). Although either H2Bub- or H3K4me-stimulated histone (poly)sumoylation blocks the recruitment of Ctk1 CTDS2 kinase, facilitating H3K36me modification for loading of the Rpd3S HDAC complex, both H3K4me and histone (poly)sumoylation are required for the chromatin binding of the Set3 HDAC complex.

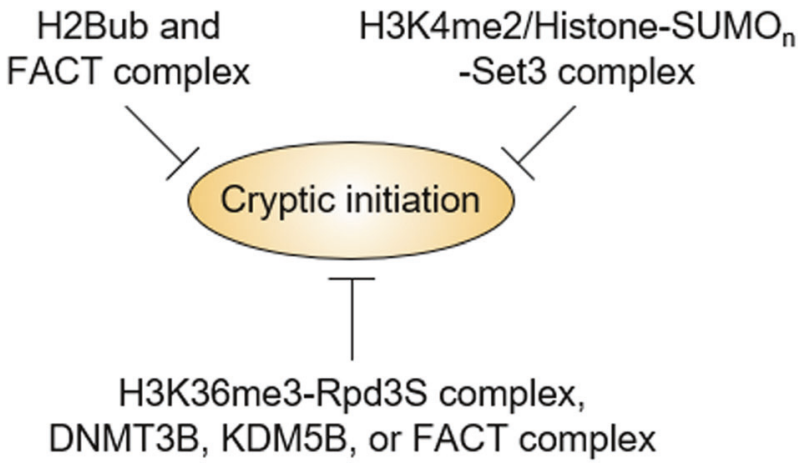

Fig. 2 Epigenetic pathways inhibiting cryptic transcription. Cryptic transcriptional initiation within the ORF is suppressed by cooperation between $\mathrm{H} 2 \mathrm{Bub}$ and the FACT complex, $\mathrm{H} 3 \mathrm{~K} 4 \mathrm{me} 2$ and the histone (poly)sumoylation-mediated Set3 HDAC complex, and the H3K36me3-dependent association of the Rpd3S complex, DNMT3B, KDM5B, or FACT complex.

reorganized, and the failure of its reassembly often leads to the exposure of cryptic promoter elements that initiate aberrant transcription from intragenic regions in a TATA-dependent or TATA-independent manner ${ }^{17}$. One such example is that cryptic transcripts are considerably increased in mutants of yeast histone chaperones Spt6, Spt16 (a subunit of the FACT complex), or Rtt106, which maintain nucleosome occupancy and DNA accessibility ${ }^{18-22}$. Moreover, following transcriptional elongation, histone acetylation should be erased to prevent cryptic transcription within the ORF region (Fig. 2). Yeast Rpd3 is the HDAC enzyme with the best-established role in inhibiting cryptic transcriptional initiation. Furthermore, Set2 histone methyltransferase-mediated H3K36me3 acts as an epigenetic mark for Rpd3S complex loading, resulting in the histone deacetylation of the ORF ${ }^{23-25}$. According to previous studies in S. cerevisiae, this Set2-Rpd3S pathway governs cryptic initiation in $\sim 30 \%$ of yeast genes, suggesting that H3K36me3-originated chromatin modifications are important for maintaining genome integrity ${ }^{26,27}$. In mammals, H3K36me3 on gene bodies also acts as a repressor of aberrant intragenic transcription by promoting the recruitment of DNMT3B DNA methyltransferase, KDM5B H3K4 demethylase, or the FACT complex ${ }^{28-31}$. The mechanisms blocking cryptic initiation in yeast share some similarities with those in mammals.

\section{H2B monoubiquitination}

Eukaryotic histones $\mathrm{H} 2 \mathrm{~A}$ and $\mathrm{H} 2 \mathrm{~B}$ are apparent targets of monoubiquitination, although $\mathrm{H} 2 \mathrm{~A}$ modification has not been detected in yeast ${ }^{32}$. Whereas a single ubiquitin moiety has been reported to be conjugated to $\mathrm{K} 119$ of $\mathrm{H} 2 \mathrm{~A}$ and $\mathrm{K} 120$ of $\mathrm{H} 2 \mathrm{~B}$ in mammals ${ }^{32}$, the site of $\mathrm{H} 2 \mathrm{Bub}$ corresponds to $\mathrm{K} 123$ in $S$. cerevisiae $^{33}, \mathrm{~K} 119$ in Schizosaccharomyces pombe $e^{34}$ and K143 in Arabidopsis $^{35}$. However, S. cerevisiae is a preferred model for studying the role and mechanism of $\mathrm{H} 2 \mathrm{Bub}$, and such studies have been extended to higher eukaryotes. Furthermore, the E2 conjugation enzymes Rad6 and the E3 ligase Bre1 mediate the $\mathrm{H} 2 \mathrm{Bub}$ process, and these enzymes are preferentially enriched across transcribed regions and correlate with transcriptional processes ${ }^{36}$. This modification is also dynamically regulated by two ubiquitin hydrolases, Ubp8 and Ubp10, which appear to regulate distinct chromatin regions ${ }^{37}$.

During the transcription cycle, diverse factors dynamically regulate the level of $\mathrm{H} 2 \mathrm{Bub}$, which also facilitates transcription activation. At the stage of transcriptional initiation, the Paf1 complex influences the interaction of Rad6 and Bre1 with RNA polymerase II (RNAPII) and subsequently favors the H2Bub step; however, the Paf1 complex does not affect the localization of Rad6 and Bre 1 in the promoter region ${ }^{38,39}$. Rad6 localization is not affected because mutations impair the association of Rad6 with elongating RNAPII in the Rtf1 subunit of the Paf1 complex. Therefore, the Paf1 complex is proposed to be required for Rad6 and Bre 1 progression during transcriptional elongation ${ }^{38}$. Other studies have shown that the Bur1/Bur2 kinase complex also affects the level of H2Bub by regulating Paf1 complex recruitment and Rad6 phosphorylation ${ }^{40,41}$.

In particular, H2Bub is closely linked to the C-terminal domain (CTD) of the largest subunit of RNAPII, which is sequentially subjected to phosphorylation at $\mathrm{S} 2$ and $\mathrm{S}^{42}$. There, while the loss of Kin28 (CDK7 in mammals), which ensures phosphorylation at CTDS5, inhibits $\mathrm{H}_{2} \mathrm{Bub}^{38}$, the removal of ubiquitin from $\mathrm{H} 2 \mathrm{~B}$ by Ubp8 allows the recruitment of Ctk1 CTDS2 kinase (P-TEFb/CDK9 in mammals) to promote the transition between the initiation and elongation steps. Hence, subsequent H3K36 methylation for binding of Rpd3S HDAC complex to gene bodies is favored ${ }^{25,43}$. Additionally, H2Bub functions cooperatively with the histone chaperone FACT complex to inhibit spurious transcriptional initiation $^{44,45}$ (Fig. 2). Therefore, such dynamic H2B ubiquitination is critical for efficient transcriptional elongation.

\section{Trans-tail regulation}

Previous studies have demonstrated that H2Bub unidirectionally facilitates the methylation of $\mathrm{K} 4$ and $\mathrm{K} 79$ at trans-histone $\mathrm{H} 3$, which is catalyzed by methyltransferases Set 1 and Dot1 in $S$. cerevisiae, respectively ${ }^{36}$. The loss of $\mathrm{H} 2 \mathrm{Bub}$ in rad $6 \Delta$, bre $1 \Delta$ or the arginine substitution mutant of $\mathrm{H} 2 \mathrm{BK} 123$ (H2BK123R) results in the abolition of $\mathrm{H} 3 \mathrm{~K} 4 \mathrm{me} 3 / \mathrm{me} 2$ and $\mathrm{H} 3 \mathrm{~K} 79 \mathrm{me} 3$ along with reductions in H3K4 monomethylation (me1) and H3K79me2 ${ }^{46,47}$, while increased levels of $\mathrm{H} 2 \mathrm{Bub}$ resulting from the loss of 
deubiquitinases Ubp8 or Ubp10 cause increases in methylation of $\mathrm{H} 3 \mathrm{~K} 4$ and $\mathrm{H} 3 \mathrm{~K} 79^{48-50}$. However, some groups suggest that the absence of $\mathrm{H} 2 \mathrm{~B}$ ubiquitination is insufficient to completely interrupt further trans-tail $\mathrm{H} 3$ methylation ${ }^{51,52}$ or that crosstalk between H2Bub and Dot1 is bidirectional in a methyltransferase activity-independent manner ${ }^{53}$.

Typically, two models have been proposed to explain the transtail regulation pathway: the wedge and bridge models ${ }^{54}$. Since ubiquitination is a bulky post-translational modification, $\mathrm{H} 2 \mathrm{Bub}$ is predicted to act as a "wedge" that opens chromatin locally, thereby allowing the access of chromatin-modifying enzymes, including histone methyltransferase ${ }^{54}$. In contrast, in the "bridge" model, H2Bub directly recruits factors for H3K4 and H3K79 methylation. However, because H2Bub does not affect the association of Set1 and Dot1 with chromatin ${ }^{55,56}, \mathrm{H} 2 \mathrm{Bub}$ is required for the recruitment of other regulatory factor(s) to mediate crosstalk. Furthermore, H2Bub facilitates the association of Swd2 with the COMPASS complex (complex of proteins associated with Set1) and triggers the recruitment of proteasomal ATPases Rpt4 and Rpt6 to chromatin, which further mediates $\mathrm{H} 3$ methylation at $\mathrm{K} 4$ and $\mathrm{K} 79^{47,57}$. Although $\mathrm{H} 2 \mathrm{Bub}$ is not required for the chromatin binding of $\mathrm{Spp}^{58}$, another subunit of COMPASS, the H2Bub-stimulated ubiquitination of Swd2subsequently recruits Spp1 for efficient H3K4 methylation ${ }^{59}$. Additionally, the presence of $\mathrm{H} 2 \mathrm{Bub}$ at the nucleosome repositions hDot $1 \mathrm{~L}$ (Dot1 in S. cerevisiae) on the nucleosomal surface, resulting in its positioning at the catalytic site of the enzyme by binding to K79 at $\mathrm{H} 3^{60}$.

On the basis of the above facts, diverse factors can affect modifications of the histone tail. For instance, mutations in the $\mathrm{H} 2 \mathrm{AN}$ terminus significantly decrease both H2Bub and H3K4 methylation without affecting the association of the modifying enzymes Rad6/Bre1 and COMPASS with chromatin ${ }^{61}$. In addition, H3R2 methylation abrogates H3K4me3 via the inhibition of Spp1 binding ${ }^{62,63}$, and this pathway is evolutionarily conserved, as H3R2 methylation by PRMT6 mutually antagonizes H3K4 methylation by the SET1/MLL (mixed lineage leukemia) complex in mammals ${ }^{64}$. In another trans-tail pathway, PRMT7-mediated H4R3 methylation interferes with the binding of the PHD finger, thereby recognizing a methylated lysine in MLL3/4 during cellular differentiation ${ }^{65,66}$.

\section{Trans-tail regulation and silencing}

The role of the trans-tail pathway was first postulated to be a regulator of transcriptional silencing because H2BK123R mutation impairs the repression of the URA3 reporter gene, located in the left-end telomere of chromosome $\mathrm{VII}^{67}$. In S. cerevisiae, there are three heterochromatin loci, subtelomeric, rDNA (rRNA-encoding DNA), and mating-type regions ${ }^{68}$, in which a characteristic pattern of histone modifications has been observed ${ }^{67,69,70}$. In particular, the Sir2 HDAC-mediated regulation of histone acetylation levels governs locus-specific chromatin condensation ${ }^{68}$. This Sir2 association is in turn tightly regulated by the trans-tail pathway ${ }^{70}$. Therefore, the loss of enzymes for H2Bub or H3K4/K79 methylation disrupts the silencing of the URA3 reporter gene at all heterochromatin $\mathrm{loci}^{70-72}$, whereas Ubp8 and Jhd2 H3K4 demethylases have an anti-silencing function in rDNA regions ${ }^{70,73}$. In addition, the modification-mediated control of Sir2 recruitment further affects cellular aging by maintaining intact telomeric chromatin $^{70,74}$.

\section{Trans-tail regulation and the Set3 pathway}

The trans-tail pathway involves not only heterochromatic silencing but also transcriptional regulation, and its cellular function in the transcription system has been intensively studied. H2Bubdependent H3K4 methylation exhibits an intrinsic gradient pattern, comprising me3 near promoters, me2 immediately downstream, and me1 in more-distal regions ${ }^{75,76}$, which is determined by the amount of time that Set 1 is tethered to RNAPII during multiple rounds of transcription ${ }^{77}$. Therefore, such patterns of H3K4 methylation suggest that this modification is positively correlated with active transcription. However, the loss of Set1 causes only minor defects in the effect of this modification during gene expression. Furthermore, a genome-wide analysis showed that only 69 and 20 transcripts were significantly increased and decreased, respectively, upon the deletion of SET1 or H3K4R mutation $^{78}$. Therefore, it is proposed that the effects of this modification on transcription are more diverse and that it regulates several functions, including learning, memory, processing, and termination ${ }^{79,80}$

Although the function of $\mathrm{H} 3 \mathrm{~K} 4 \mathrm{me} 3$ in transcription has been comparatively well studied, that of $\mathrm{H} 3 \mathrm{~K} 4 \mathrm{me} 2$ remains unclear. However, Buratowski's group showed that H3K4me2 has distinct effects on the transcription cycle a decade ago ${ }^{13}$. The expression of Set1 lacking the RRM (RNA Recognition Motif) domain, which eliminates $\mathrm{H} 3 \mathrm{~K} 4 \mathrm{me} 3$ but has no effect on $\mathrm{H} 3 \mathrm{~K} 4 \mathrm{me} 2^{72}$, causes no apparent increase in histone acetylation in $5^{\prime}$ transcribed regions, suggesting that $\mathrm{H} 3 \mathrm{~K} 4 \mathrm{me} 2$ is sufficient to suppress histone acetylation in the $5^{\prime}$ ORF region ${ }^{13}$. Furthermore, among several candidate proteins, including proteins with the PHD domain that binds methylated $\mathrm{H} 3 \mathrm{~K} 4$ in vitro ${ }^{81}$, the Set3 protein preferentially binds H3K4me2 peptides. Subsequently, H3K4me2 influences the association of the Set3 complex, including two active HDAC subunits, Hos2 and Hst1, with chromatin ${ }^{13}$. The loss of such subunits and additional accessory proteins, Sif2 and Snt1, in the Set3 complex increases histone acetylation at $5^{\prime}$ ORF loci $^{13}$. Therefore, the Set3 complex-mediated deacetylation of histones in $5^{\prime}$ ORFs represses the cryptic initiation of both sense and antisense transcription in gene bodies ${ }^{14}$. Taken together, these findings indicate that $\mathrm{H} 2 \mathrm{Bub}$ and the subsequent $\mathrm{H} 3 \mathrm{~K} 4 \mathrm{me}$ 2-driven histone deacetylation process maintain transcriptional fidelity by suppressing spurious transcriptional initiation.

\section{Trans-tail regulation and histone sumoylation}

Recent findings revealed that histone sumoylation is also closely related to this trans-tail regulation. Histones are an evolutionarily conserved target of small ubiquitin-like modifier (SUMO) modification, which is sequentially carried out by the following enzymes: heterodimeric Aos1/Uba2 (SAE1/SAE2 in mammals), SUMO-activating enzyme (E1), Ubc9 SUMO-conjugating enzyme (E2), and several SUMO ligases (E3s) ${ }^{12,82}$. Since the first reports of human histone-SUMO conjugates in $2003^{83}$, several sumoylation sites on four core histones and histone variants have been discovered in human cells and S. cerevisiae ${ }^{12}$. In the case of histone variants, the sumoylation of the $\mathrm{H} 3$ variant $\mathrm{Cse} 4$ mediates its proper localization at the centromere ${ }^{84-86}$, and the repair of DNA double-strand breaks is affected by H2A.Z sumoylation ${ }^{87}$. Until recently, the effect of SUMO on core histones was assumed to be transcriptional repression via the inhibition of, or competition with, gene activation markers such as monoubiquitination and acetylation on histones or the recruitment of their enzymes ${ }^{12}$.This type of modification-dependent control is not simple, and it contributes to the remarkably complex transcription program.

The correlation between the trans-tail pathway and histone sumoylation was incidentally discovered in a functional study of the Ulp2 protease ${ }^{88}$, which efficiently disassembles poly-SUMO chains on proteins and acts as an essential regulator of cell homeostasis in S. cerevisiae $89-92$. Ulp2 is preferentially associated with constitutive genes, and its loss impedes efficient gene expression via a defect in RNAPII recruitment ${ }^{88}$. Genetic interactions of ULP2 with genes encoding H2Bub enzymes, RAD6 and $B R E 1$, were first discovered in synthetic lethal screening assays, implying that these genes are involved in similar pathways ${ }^{88}$. Furthermore, H2Bub is required for the histone sumoylation and chromatin localization of Ulp2, allowing subsequent histone desumoylation steps during transcription ${ }^{88}$. Furthermore, persistent polySUMO conjugation to H2B or Ulp2 loss blocks Ctk1 


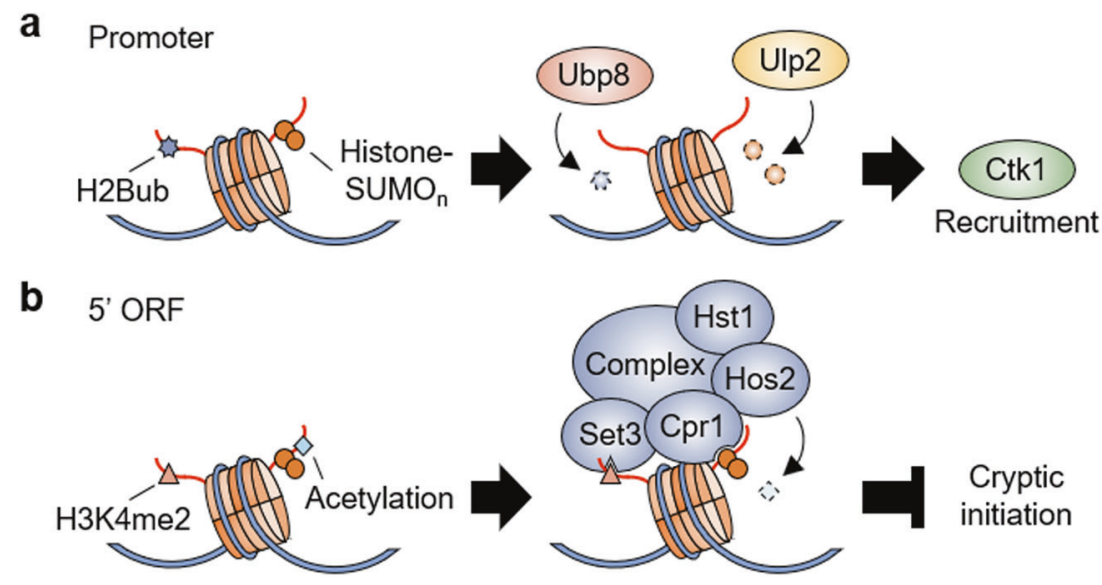

Fig. 3 Dual functions of histone sumoylation in transcription. a In the promoter region, the elimination of ubiquitin and SUMO from histones by Ubp8 and Ulp2, respectively, recruits Ctk1 to chromatin to facilitate the transition between transcriptional initiation and elongation. $\mathbf{b}$ In the $5^{\prime}$ ORF region, $\mathrm{H} 3 \mathrm{~K} 4 \mathrm{me} 2$ and histone (poly)sumoylation individually provide binding platforms for Set3 and Cpr1 in the Set3 complex, including two HDAC enzymes, Hst1 and Hos2, to suppress internal cryptic initiation.

nucleosome binding, thus limiting CTDS2 phosphorylation and efficient transcriptional elongation ${ }^{88}$. Such defects in Ctk1 recruitment are similarly observed in cells lacking Ubp $8^{43}$, suggesting that both ubiquitin and SUMO conjugation to histones serve to modulate the level of CTDS2 phosphorylation required for the efficient transition between transcriptional initiation and elongation steps (Fig. 3a).

Similar to the effect of H2Bub on histone sumoylation, H3K4me2, but not me3, is a prerequisite step for histone sumoylation to occur, and this pathway is unidirectional and not bidirectional ${ }^{93}$. Additionally, a histone $\mathrm{H} 2 \mathrm{~B}$ mutation causing defective $\mathrm{H} 2 \mathrm{~B}$ sumoylation impairs the association of two subunits of the Set3 complex, Set3 and Hos2, with target genes. This process results in hyper-histone acetylation, and shows strong sensitivity to 6azauracil, which is a general indicator employed for evaluating transcriptional elongation ${ }^{94}$, at $34^{\circ} \mathrm{C}^{93}$. Another subunit $\mathrm{Cpr} 1$ of the Set3 complex recognizes SUMO-conjugated histones via its SIM (SUMO-interacting motif) and promotes Set3 complex loading onto nucleosomes ${ }^{93}$. Notably, such H2B mutation impedes the recruitment of the Set3 complex to ncRNA (noncoding RNA) genes as well as protein-coding genes, which greatly increases the transcription of ncRNAs from internal sites within $\mathrm{ORFs}^{93}$. Therefore, an elaborate histone modification network involving the consecutive ubiquitination, methylation, sumoylation and deacetylation of histones promotes transcriptional elongation by suppressing cryptic intragenic initiation (Fig. 3b).

\section{CONCLUDING REMARKS}

The effect of H2Bub on $\mathrm{H} 3 \mathrm{~K} 4$ and $\mathrm{H} 3 \mathrm{~K} 79$ methylation itself has been well characterized, and this trans-tail regulation pathway is clearly linked with chromatin dynamics and diverse nuclear functions ${ }^{36}$. However, the ultimate role of this crosstalk pathway in transcription has remained unclear until recently. Here, we have briefly reported that the transcriptional mechanism is elaborately regulated by the $\mathrm{H} 2 \mathrm{Bub}$-originated regulation pathway. In an early transcription stage, the CTDS5-phosphorylated form of RNAPII and the Paf1 complex are both required for Rad6 and Bre1-mediated $\mathrm{H}_{2} \mathrm{Bub}^{38,39}$, resulting in two sequential histone modifications, Set1-mediated H3K4 methylation and histone sumoylation by Ubc9 and E3 ligase(s) ${ }^{46,47,88,93}$. However, both H2Bub and histone sumoylation act as barriers to Ctk1-dependent CTDS2 phosphorylation, which then favors the removal of ubiquitin and SUMO proteins from histones catalyzed by Ubp8 and Ulp2, respectively. This process subsequently facilitates CTDS2 phosphorylation by $\mathrm{Ctk} 1^{43,88,93}$. During transcriptional elongation, H2Bub and histone sumoylation cycles are repeated ${ }^{43,88,93}$, while an $\mathrm{H} 3 \mathrm{~K} 4$ methylation gradient in which $\mathrm{H} 3 \mathrm{~K} 4 \mathrm{me} 3$ in the promoter and $\mathrm{H} 3 \mathrm{~K} 4 \mathrm{me} 2$ occurs in the $5^{\prime}$ ORF is gradually established ${ }^{76,77}$. In further steps, the Set3 and Cpr1 subunits of the Set3 HDAC complex recognize H3K4me2 and histone sumoylation, respectively, which then facilitates the deacetylation of histones in $5^{\prime}$ ORF regions to prevent cryptic internal initiation ${ }^{13,88,93}$.

It remains unclear whether the trans-tail pathway-mediated suppression of spurious transcription is evolutionarily conserved in mammals. However, the mammalian ortholog of Set3 is MLL5, as suggested by their sequence similarities. Both proteins also lack intrinsic methyltransferase activity ${ }^{95}$. Furthermore, MLL5 is a component of the NCoR-SMRT complex, which acts as a corepressor of hormone receptors and recruits HDAC to regulate gene expression ${ }^{96}$. Moreover, despite the functional correlation between the Set3 complex and NCOR-SMRT, the role of MLL5 in the inhibition of cryptic transcription has not yet been determined. Although Dot1dependent $\mathrm{H} 3 \mathrm{~K} 79$ methylation is involved in active transcription and genome stability ${ }^{97}$, no evidence related to cryptic initiation has been reported. In addition, the effect of histone sumoylation on the Set3 complex has been studied only in S. cerevisiae ${ }^{93}$, and functional studies on histone sumoylation in higher eukaryotes are required to understand its complex mechanism.

On the basis of the above findings, the misregulation of $\mathrm{H} 2 \mathrm{Bub}$ or histone methylation has been associated with several human diseases, including cancers and neurodegenerative disorders ${ }^{98,99}$. Therefore, although there are no available reports of the effects of cryptic transcription inhibited by trans-tail regulation in human diseases, many diseases are known to be closely correlated with aberrant expression of ncRNAs, which are key factors in gene expression control, genome stability, and chromatin dynamics ${ }^{100}$. Hence, the better characterization of how epigenetic regulation modulates the monitoring mechanisms of spurious transcription may be a promising avenue for future research to develop new therapies for various disorders.

\section{REFERENCES}

1. Kornberg, R. D. \& Lorch, Y. L. Twenty-five years of the nucleosome, fundamental particle of the eukaryote chromosome. Cell 98, 285-294 (1999).

2. Kouzarides, T. Chromatin modifications and their function. Cell 128, 693-705 (2007).

3. Kothapalli, N. et al. Biological functions of biotinylated histones. J. Nutr. Biochem. 16, 446-448 (2005).

4. Ruthenburg, A. J., Li, H., Patel, D. J. \& Allis, C. D. Multivalent engagement of chromatin modifications by linked binding modules. Nat. Rev. Mol. Cell Biol. 8, 983-994 (2007). 
5. Lee, J. S., Smith, E. \& Shilatifard, A. The language of histone crosstalk. Cell 142 682-685 (2010).

6. Zippo, A. et al. Histone crosstalk between H3S10ph and H4K16ac generates a histone code that mediates transcription elongation. Cell 138, 1122-1136 (2009).

7. Shimada, M. et al. Chk1 is a histone $\mathrm{H} 3$ threonine 11 kinase that regulates DNA damage-induced transcriptional repression. Cell 132, 221-232 (2008).

8. Ringel, A. E., Cieniewicz, A. M., Taverna, S. D. \& Wolberger, C. Nucleosome competition reveals processive acetylation by the SAGA HAT module. Proc. Natl Acad. Sci. USA 112, E5461-E5470 (2015).

9. Govind, C. K., Zhang, F., Qiu, H. F., Hofmeyer, K. \& Hinnebusch, A. G. Gen5 promotes acetylation, eviction, and methylation of nucleosomes in transcribed coding regions. Mol. Cell 25, 31-42 (2007).

10. Werner, M. \& Ruthenburg, A. J. The United States of Histone Ubiquitylation and Methylation. Mol. Cell 43, 5-7 (2011).

11. Chandrasekharan, M. B., Huang, F. \& Sun, Z. W. Histone H2B ubiquitination and beyond Regulation of nucleosome stability, chromatin dynamics and the transhistone H3 methylation. Epigenetics 5, 460-468 (2010).

12. Ryu, H. Y. \& Hochstrasser, M. Histone sumoylation and chromatin dynamics. Nucleic Acids Res. 49, 6043-6052 (2021).

13. Kim, T. \& Buratowski, S. Dimethylation of H3K4 by Set 1 recruits the Set3 histone deacetylase complex to $5^{\prime}$ transcribed regions. Cell 137, 259-272 (2009).

14. Kim, T., Xu, Z., Clauder-Münster, S., Steinmetz, L. M. \& Buratowski, S. Set3 HDAC mediates effects of overlapping noncoding transcription on gene induction kinetics. Cell 150, 1158-1169 (2012).

15. Li, B., Carey, M. \& Workman, J. L. The role of chromatin during transcription. Cell 128, 707-719 (2007).

16. Clapier, C. R. \& Cairns, B. R. The biology of chromatin remodeling complexes. Annu. Rev. Biochem. 78, 273-304 (2009).

17. Smolle, M. \& Workman, J. L. Transcription-associated histone modifications and cryptic transcription. Biochim. Biophys. Acta Gene Regul. Mech. 1829, 84-97 (2013).

18. Kaplan, C. D., Laprade, L. \& Winston, F. Transcription elongation factors repress transcription initiation from cryptic sites. Science 301, 1096-1099 (2003).

19. Mason, P. B. \& Struhl, K. The FACT complex travels with elongating RNA polymerase II and is important for the fidelity of transcriptional initiation in vivo. Mol. Cell. Biol. 23, 8323-8333 (2003).

20. Stevens, J. R. et al. FACT, the Bur kinase pathway, and the histone co-repressor HirC have overlapping nucleosome-related roles in yeast transcription elongation. PLOS ONE 6, e25644 (2011).

21. Silva, A. C. et al. The replication-independent histone $\mathrm{H} 3-\mathrm{H} 4$ chaperones $\mathrm{HIR}_{t}$ ASF1, and RTT106 co-operate to maintain promoter fidelity. J. Biol. Chem. 287, 1709-1718 (2012)

22. Imbeault, D., Gamar, L., Rufiange, A., Paquet, E. \& Nourani, A. The Rtt106 histone chaperone is functionally linked to transcription elongation and is involved in the regulation of spurious transcription from cryptic promoters in yeast. J. Biol. Chem. 283, 27350-27354 (2008).

23. Carrozza, M. J. et al. Histone H3 methylation by Set2 directs deacetylation of coding regions by Rpd3S to suppress spurious intragenic transcription. Cell 123, 581-592 (2005).

24. Joshi, A. A. \& Struhl, K. Eaf3 chromodomain interaction with methylated H3K36 links histone deacetylation to Pol II elongation. Mol. Cell 20, 971-978 (2005).

25. Keogh, M.-C. et al. Cotranscriptional set2 methylation of histone $\mathrm{H} 3$ lysine 36 recruits a repressive Rpd3 complex. Cell 123, 593-606 (2005).

26. Li, B. et al. Combined action of PHD and chromo domains directs the Rpd3S HDAC to transcribed chromatin. Science 316, 1050-1054 (2007).

27. Li, B. et al. Infrequently transcribed long genes depend on the Set2/Rpd3S pathway for accurate transcription. Genes Dev. 21, 1422-1430 (2007).

28. Teissandier, A. \& Bourc'his, D. Gene body DNA methylation conspires with H3K36me3 to preclude aberrant transcription. EMBO J. 36, 1471-1473 (2017).

29. Carvalho, S. et al. Histone methyltransferase SETD2 coordinates FACT recruitment with nucleosome dynamics during transcription. Nucleic Acids Res. 41, 2881-2893 (2013).

30. Neri, F. et al. Intragenic DNA methylation prevents spurious transcription initiation. Nature 543, 72-77 (2017).

31. Xie, L. et al. KDM5B regulates embryonic stem cell self-renewal and represses cryptic intragenic transcription. EMBO J. 30, 1473-1484 (2011).

32. Osley, M. A. Regulation of histone $\mathrm{H} 2 \mathrm{~A}$ and $\mathrm{H} 2 \mathrm{~B}$ ubiquitylation. Brief. Funct. Genom. 5, 179-189 (2006).

33. Robzyk, K., Recht, L. \& Osley, M. A. Rad6-dependent ubiquitination of histone H2B in yeast. Science 287, 501-504 (2000).

34. Tanny, J. C., Erdjument-Bromage, H., Tempst, P. \& Allis, C. D. Ubiquitylation of histone $\mathrm{H} 2 \mathrm{~B}$ controls RNA polymerase II transcription elongation independently of histone H3 methylation. Genes Dev. 21, 835-847 (2007).
35. Sridhar, V. V. et al. Control of DNA methylation and heterochromatic silencing by histone H2B deubiquitination. Nature 447, 735-738 (2007).

36. Weake, V. M. \& Workman, J. L. Histone ubiquitination: Triggering gene activity. Mol. Cell 29, 653-663 (2008).

37. Schulze, J. M. et al. Splitting the task: Ubp8 and Ubp10 deubiquitinate different cellular pools of H2BK123. Genes Dev. 25, 2242-2247 (2011).

38. Xiao, $\mathrm{T}$. et al. Histone $\mathrm{H} 2 \mathrm{~B}$ ubiquitylation is associated with elongating RNA polymerase II. Mol. Cell. Biol. 25, 637-651 (2005).

39. Wood, A., Schneider, J., Dover, J., Johnston, M. \& Shilatifard, A. The Paf1 complex is essential for histone monoubiquitination by the Rad6-Bre1 complex, which signals for histone methylation by COMPASS and Dot1p. J. Biol. Chem. 278, 34739-34742 (2003).

40. Laribee, R. N. et al. BUR kinase selectively regulates $\mathrm{H} 3 \mathrm{~K} 4$ trimethylation and $\mathrm{H} 2 \mathrm{~B}$ ubiquitylation through recruitment of the PAF elongation complex. Curr. Biol. 15, 1487-1493 (2005).

41. Wood, A., Schneider, J., Dover, J., Johnston, M. \& Shilatifard, A. The Bur1/Bur2 complex is required for histone $\mathrm{H} 2 \mathrm{~B}$ monoubiquitination by Rad6/Bre1 and histone methylation by COMPASS. Mol. Cell 20, 589-599 (2005).

42. Hartzog, G. A. \& Tamkun, J. W. A new role for histone tail modifications in transcription elongation. Genes Dev. 21, 3209-3213 (2007).

43. Wyce, A. et al. H2B ubiquitylation acts as a barrier to Ctk1 nucleosomal recruitment prior to removal by Ubp8 within a SAGA-related complex. Mol. Cell 27, 275-288 (2007).

44. Laribee, R. N., Fuchs, S. M. \& Strahl, B. D. H2B ubiquitylation in transcriptional control: a FACT-finding mission. Genes Dev. 21, 737-743 (2007).

45. Pavri, R. et al. Histone $\mathrm{H} 2 \mathrm{~B}$ monoubiquitination functions cooperatively with FACT to regulate elongation by RNA polymerase II. Cell 125, 703-717 (2006).

46. Nakanishi, S. et al. Histone $\mathrm{H} 2 \mathrm{BK} 123$ monoubiquitination is the critical determinant for H3K4 and H3K79 trimethylation by COMPASS and Dot1. J. Cell Biol. 186, 371-377 (2009).

47. Lee, J. S. et al. Histone crosstalk between $\mathrm{H} 2 \mathrm{~B}$ monoubiquitination and $\mathrm{H} 3$ methylation mediated by COMPASS. Cell 131, 1084-1096 (2007).

48. Henry, K. W. et al. Transcriptional activation via sequential histone $\mathrm{H} 2 \mathrm{~B}$ ubiquitylation and deubiquitylation, mediated by SAGA-associated Ubp8. Genes Dev. 17, 2648-2663 (2003).

49. Daniel, J. A. et al. Deubiquitination of histone H2B by a yeast acetyltransferase complex regulates transcription. J. Biol. Chem. 279, 1867-1871 (2004).

50. Gardner, R. G., Nelson, Z. W. \& Gottschling, D. E. Ubp10/Dot4p regulates the persistence of ubiquitinated histone $\mathrm{H} 2 \mathrm{~B}$ : Distinct roles in telomeric silencing and general chromatin. Mol. Cell. Biol. 25, 6123-6139 (2005).

51. Foster, E. R. \& Downs, J. A. Methylation of H3 K4 and K79 is not strictly dependent on H2B K123 ubiquitylation. J. Biol. Chem. 184, 631-638 (2009).

52. Dehe, $\mathrm{P}$. M. et al. Histone $\mathrm{H} 3$ lysine 4 mono-methylation does not require ubiquitination of histone H2B. J. Mol. Biol. 353, 477-484 (2005).

53. van Welsem, T. et al. Dot1 promotes $\mathrm{H} 2 \mathrm{~B}$ ubiquitination by a methyltransferaseindependent mechanism. Nucleic Acids Res. 46, 11251-11261 (2018).

54. Henry, K. W. \& Berger, S. L. Trans-tail histone modifications: wedge or bridge? Nat. Struct. Mol. Biol. 9, 565-566 (2002).

55. Ng, H. H., Robert, F., Young, R. A. \& Struhl, K. Targeted recruitment of Set1 histone methylase by elongating Pol II provides a localized mark and memory of recent transcriptional activity. Mol. Cell 11, 709-719 (2003).

56. Shahbazian, M. D., Zhang, K. L. \& Grunstein, M. Histone H2B ubiquitylation controls processive methylation but not monomethylation by Dot1 and Set1. Mol. Cell 19, 271-277 (2005)

57. Ezhkova, E. \& Tansey, W. P. Proteasomal ATPases link ubiquitylation of histone $\mathrm{H} 2 \mathrm{~B}$ to methylation of histone H3. Mol. Cell 13, 435-442 (2004).

58. Takahashi, Y. H. et al. Regulation of H3K4 trimethylation via Cps40 (Spp1) of COMPASS Is monoubiquitination independent: implication for a Phe/Tyr switch by the catalytic domain of Set1. Mol. Cell. Biol. 29, 3478-3486 (2009).

59. Vitaliano-Prunier, A. et al. Ubiquitylation of the COMPASS component Swd2 links H2B ubiquitylation to H3K4 trimethylation. Nat. Cell Biol. 10, 1365-1371 (2008).

60. Zhou, L. J. et al. Evidence that ubiquitylated H2B corrals hDot1L on the nucleosomal surface to induce H3K79 methylation. Nat. Commun. 7, 10589 (2016).

61. Zheng, S. T., Wyrick, J. J. \& Reese, J. C. Novel trans-Tail Regulation of H2B Ubiquitylation and H3K4 Methylation by the N Terminus of Histone H2A. Mol. Cell. Biol. 30, 3635-3645 (2010).

62. Kirmizis, A. et al. Arginine methylation at histone H3R2 controls deposition of H3K4 trimethylation. Nature 449, 928-932 (2007).

63. Yuan, C. C. et al. Histone H3R2 symmetric dimethylation and histone H3K4 trimethylation are tightly correlated in eukaryotic genomes. Cell Rep. 1, 83-90 (2012).

64. Guccione, E. et al. Methylation of histone H3R2 by PRMT6 and H3K4 by an MLL complex are mutually exclusive. Nature 449, 933-937 (2007). 
65. Dhar, S. S. et al. Trans-tail regulation of MLL4-catalyzed H3K4 methylation by H4R3 symmetric dimethylation is mediated by a tandem PHD of MLL4. Genes Dev. 26, 2749-2762 (2012).

66. Liu, Y. L. et al. Structural insights into trans-histone regulation of H3K4 methylation by unique histone H4 binding of MLL3/4. Nat. Commun. 10, 36 (2019).

67. Sun, Z. W. \& Allis, C. D. Ubiquitination of histone $\mathrm{H} 2 \mathrm{~B}$ regulates $\mathrm{H} 3$ methylation and gene silencing in yeast. Nature 418, 104-108 (2002).

68. Grunstein, M. Yeast heterochromatin: regulation of its assembly and inheritance by histones. Cell 93, 325-328 (1998).

69. Emre, N. C. et al. Maintenance of low histone ubiquitylation by Ubp10 correlates with telomere-proximal Sir2 association and gene silencing. Mol. Cell 17, 585-594 (2005).

70. Rhie, B. H., Song, Y. H., Ryu, H. Y. \& Ahn, S. H. Cellular aging is associated with increased ubiquitylation of histone $\mathrm{H} 2 \mathrm{~B}$ in yeast telomeric heterochromatin. Biochem. Biophys. Res. Commun. 439, 570-575 (2013).

71. Singer, M. S. et al. Identification of high-copy disruptors of telomeric silencing in Saccharomyces cerevisiae. Genetics 150, 613-632 (1998).

72. Fingerman, I. M., Wu, C.-L., Wilson, B. D. \& Briggs, S. D. Global loss of Set1mediated H3 Lys4 trimethylation is associated with silencing defects in Saccharomyces cerevisiae. J. Biol. Chem. 280, 28761-28765 (2005).

73. Ryu, H. Y. \& Ahn, S. Yeast histone H3 lysine 4 demethylase Jhd 2 regulates mitotic rDNA condensation. BMC Biol. 12, 75 (2014).

74. Dang, W. et al. Histone $\mathrm{H} 4$ lysine 16 acetylation regulates cellular lifespan. Nature 459, 802-807 (2009).

75. Barski, A. et al. High-resolution profiling of histone methylations in the human genome. Cell 129, 823-837 (2007).

76. Pokholok, D. K. et al. Genome-wide map of nucleosome acetylation and methylation in yeast. Cell 122, 517-527 (2005).

77. Soares, L. M. et al. Determinants of histone H3K4 methylation patterns. Mol. Cell 68, 773-785.e6 (2017).

78. Margaritis, T. et al. Two distinct repressive mechanisms for histone 3 lysine 4 methylation through promoting 3 '-end antisense transcription. PLoS Genet. 8, e1002952 (2012).

79. Howe, F. S., Fischl, H., Murray, S. C. \& Mellor, J. Is H3K4me3 instructive for transcription activation. BioEssays 39, 1-12 (2017).

80. Collins, B. E., Greer, C. B., Coleman, B. C. \& Sweatt, J. D. Histone H3 lysine K4 methylation and its role in learning and memory. Epigenetics Chromatin 12, 7 (2019).

81. Shi, X. B. et al. Proteome-wide analysis in Saccharomyces cerevisiae identifies several PHD fingers as novel direct and selective binding modules of histone $\mathrm{H} 3$ methylated at either lysine 4 or lysine 36. J. Biol. Chem. 282, 2450-2455 (2007).

82. Hendriks, I. A. \& Vertegaal, A. C. A comprehensive compilation of SUMO proteomics. Nat. Rev. Mol. Cell Biol. 17, 581-595 (2016).

83. Shiio, Y. \& Eisenman, R. N. Histone sumoylation is associated with transcriptional repression. Proc. Natl Acad. Sci. U. S. A. 100, 13225-13230 (2003).

84. Ohkuni, K. et al. SUMO-targeted ubiquitin ligase (STUbL) SIx5 regulates proteolysis of centromeric histone $\mathrm{H} 3$ variant $\mathrm{Cse} 4$ and prevents its mislocalization to euchromatin. Mol. Biol. Cell. 27, 1500-1510 (2016).

85. Ohkuni, K. et al. $\mathrm{N}$-terminal sumoylation of centromeric histone $\mathrm{H} 3$ variant $\mathrm{Cse} 4$ regulates its proteolysis to prevent mislocalization to non-centromeric chromatin. G3 (Bethesda) 8, 1215-1223 (2018).

86. Ohkuni, K. et al. Deposition of centromeric histone H3 Variant CENP-A/Cse4 into chromatin is facilitated by Its C-terminal sumoylation. Genetics 214, 839-854 (2020).

87. Kalocsay, M., Hiller, N. J. \& Jentsch, S. Chromosome-wide Rad51 spreading and SUMO-H2A.Z-dependent chromosome fixation in response to a persistent DNA double-strand break. Mol. Cell 33, 335-343 (2009).

88. Ryu, H. Y. et al. The Ulp2 SUMO protease promotes transcription elongation through regulation of histone sumoylation. EMBO J. 38, e102003 (2019).

89. Bylebyl, G. R., Belichenko, I. \& Johnson, E. S. The SUMO isopeptidase Ulp2 prevents accumulation of SUMO chains in yeast. J. Biol. Chem. 278, 44113-44120 (2003).

90. Ryu, H. Y., Ahn, S. H. \& Hochstrasser, M. SUMO and cellular adaptive mechanisms. Exp. Mol. Med. 52, 931-939 (2020).
91. Ryu, H. Y. et al. Distinct adaptive mechanisms drive recovery from aneuploidy caused by loss of the Ulp2 SUMO protease. Nat. Commun. 9, 5417 (2018).

92. Ryu, H. Y., Wilson, N. R., Mehta, S., Hwang, S. S. \& Hochstrasser, M. Loss of the SUMO protease Ulp2 triggers a specific multichromosome aneuploidy. Genes Dev. 30, 1881-1894 (2016).

93. Ryu, H. Y., Zhao, D., Li, J., Su, D. \& Hochstrasser, M. Histone sumoylation promotes Set3 histone-deacetylase complex-mediated transcriptional regulation. Nucleic Acids Res. 48, 12151-12168 (2020).

94. Conaway, J. W., Shilatifard, A., Dvir, A. \& Conaway, R. C. Control of elongation by RNA polymerase II. Trends Biochem. Sci. 25, 375-380 (2000).

95. Mas-Y-Mas, S. et al. The human mixed lineage leukemia 5 (mll5), a sequentially and structurally divergent set domain-containing protein with no intrinsic catalytic activity. PLoS ONE 11, e0165139 (2016).

96. Ng, H. H. \& Bird, A. Histone deacetylases: silencers for hire. Trends Biochem. Sci. 25, 121-126 (2000)

97. Wood, K., Tellier, M. \& Murphy, S. DOT1L and H3K79 methylation in transcription and genomic stability. Biomolecules 8, 11 (2018).

98. Greer, E. L. \& Shi, Y. Histone methylation: a dynamic mark in health, disease and inheritance. Nat. Rev. Genet. 13, 343-357 (2012).

99. Jeusset, L. M. P. \& McManus, K. J. Developing targeted therapies that exploit aberrant histone ubiquitination in cancer. Cells 8, 165 (2019).

100. Wery, M., Kwapisz, M. \& Morillon, A. Noncoding RNAs in gene regulation. Wiley Interdiscip. Rev. Syst. Biol. Med. 3, 728-738 (2011).

\section{ACKNOWLEDGEMENTS}

This study was supported by the National Research Foundation of Korea (NRF) grants funded by the South Korean government (MSIT) (2020R1C1C1009367, 2020R1A4A10 18280, and 2020R1F1A1076705). We thank Junho Song for comments on the manuscript.

\section{COMPETING INTERESTS}

The authors declare no competing interests.

\section{ADDITIONAL INFORMATION}

Correspondence and requests for materials should be addressed to Jungmin Choi or Hong-Yeoul Ryu.

Reprints and permission information is available at http://www.nature.com/ reprints

Publisher's note Springer Nature remains neutral with regard to jurisdictional claims in published maps and institutional affiliations.

Open Access This article is licensed under a Creative Commons Attribution 4.0 International License, which permits use, sharing, adaptation, distribution and reproduction in any medium or format, as long as you give appropriate credit to the original author(s) and the source, provide a link to the Creative Commons license, and indicate if changes were made. The images or other third party material in this article are included in the article's Creative Commons license, unless indicated otherwise in a credit line to the material. If material is not included in the article's Creative Commons license and your intended use is not permitted by statutory regulation or exceeds the permitted use, you will need to obtain permission directly from the copyright holder. To view a copy of this license, visit http://creativecommons. org/licenses/by/4.0/

(c) The Author(s) 2021 